\title{
Lineshapes, shifts and broadenings in dynamical X-ray photoelectron spectroscopy
}

\author{
Aykutlu Dâna* \\ UNAM Institute of Materials Science and Nanotechnology, Bilkent University, 06800 Ankara, Turkey
}

\section{A R T I C L E I N F O}

\section{Article history:}

Available online 21 October 2009

\begin{abstract}
A B S T R A C T
We describe in detail a model that can be used to estimate the X-ray photoelectron spectroscopic data of surfaces when a time varying bias or a modulation of the electrical properties of the surface is applied by external stimulation, in the presence of a neutralizing electron beam. Using the model and spectra recorded under periodic sample bias modulation, certain electronic properties related to charging dynamics of the surface can be estimated. The resulting technique is a non-contact impedance measurement technique with chemical specificity. Typical behavior of spectra under a square wave bias is given. Alternative modulation schemes are investigated, including small-signal square wave modulation, sinusoidal modulation and modulation of sample resistivity under fixed bias.
\end{abstract}

(c) 2009 Elsevier B.V. All rights reserved.

\section{Introduction}

$\mathrm{X}$-ray photoelectron spectroscopy (XPS) is a celebrated technique $[1,2]$, that can be used to study composition and structures of surfaces and nanostructures on surfaces. Specifically, XPS spectra contain information about the chemical bonding states of the atoms under inspection [3,4] and structural information about the sample can be deduced from such data. The differences between the binding energies due to different bonding configurations of a species are typically on the order of an $\mathrm{eV}$, and these shifts can be well resolved with spectroscopic systems with instrumental energy resolutions on the order of tens of meV. The inherent width of peaks are generally greater than the instrumental resolution and data processing is employed to estimate peak positions and separate multiple peaks corresponding to different bonding states. The chemical shifts of species can be calculated using ab initio methods [5-7] for different materials and structures. However, charging effects [8] and differential charging effects reduce the reliability of data obtained for samples where there is a finite conductance from the surface to the ground plane. Moreover, for novel technological applications, it may be desirable to study samples where a finite impedance to the substrate is an integral part of the sample system to be studied [9]. Therefore, a description of the XPS spectra that takes into account the variations of the surface potential due to local electrical properties of the sample is useful in more reliable interpretation of data. By applying voltage stress to the sample rod while recording XPS spectra, it has been shown that the extent of charging can be

\footnotetext{
* Tel.: +90 312 2903502; fax: +90 3122664365

E-mail address: aykutlu@unam.bilkent.edu.tr.
}

controlled via which a range of analytical and electrical information could be extracted [10-13]. In addition to static information derived from application of DC voltage stress, dynamical information can also be extracted if the voltage stimuli is applied in the form of pulses, as recently reported. Since an electron and/or an ion source can not be turned on and off rapidly, the application of the voltage stress to the sample rod brings an important advantage for dynamical measurements [14-16].

In this article, we describe a theoretical model that can be used to include the effect of local charging to the XPS spectra. The model assumes that an electron gun supplies a surface potential dependent current to the sample. Such charge neutralization schemes are used to compensate the deficiency of electrons created by photoemission and eliminate the surface potential shifts to obtain more reliable spectra $[17,18]$. The model is a dynamic model, meaning, time dependent surface potentials can be interpreted using the model and resulting XPS spectra can be estimated. In the simplest case, characteristic charging time constants can be deduced using the model along with the finite resistance from the surface to the substrate. The model can be used to study other time dependent or transient phenomena that can effect the surface potential, such as modification of XPS spectra under time varying illumination. The model can be extended, for example, using angle dependent collection of photoelectrons to depth dependent electrical characterization with chemical specificity. Higher spatial resolution can be achieved using focused X-ray illumination.

\section{Surface charging model under electron beam compensation}

The simplified surface charging model used in this article is illustrated in Fig. 1a and b. Previously, the model was demon- 
(a) monochromatic X-Rays
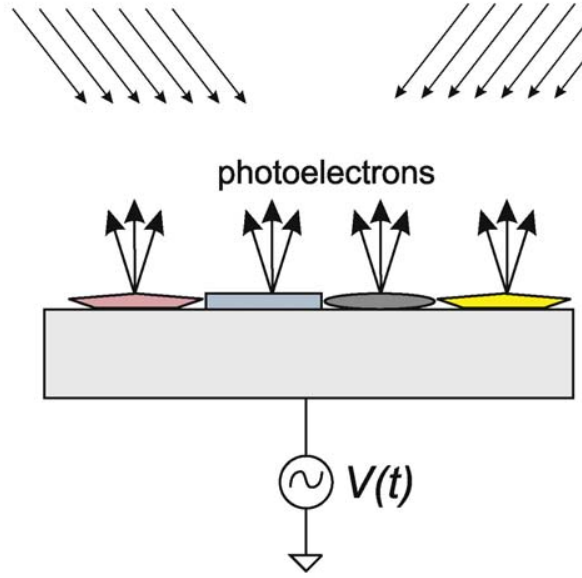

(b)

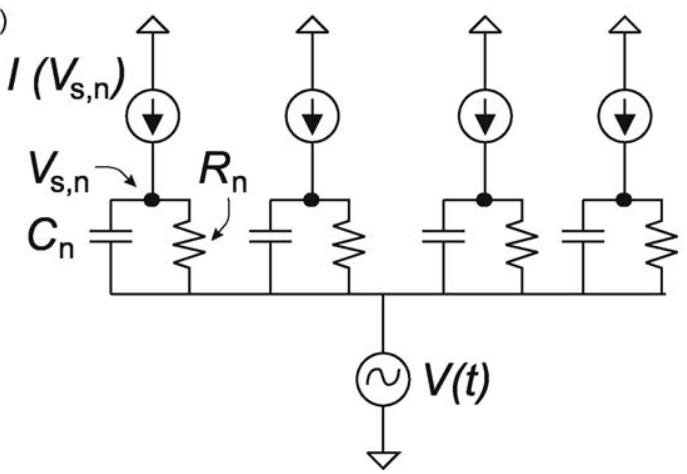

Fig. 1. (a) Schematic description of the dynamic XPS experiment where the sample is illuminated with X-rays and a charge compensating low energy electron beam. Different domains are denoted by different geometric shapes and colors. The sample substrate is subjected to a time varying external stimulus $V(t)$. (b) The surface potential of each domain is described by a lumped $R$ and $C$ circuit, where the $\mathrm{X}$-ray-induced photoemission currents and electron gun currents are lumped into a single voltage controlled current source. The surface of the domains are assumed to be electrically isolated.

strated to accurately estimate XPS spectra of samples subject to external voltage modulation [19-22]. In the dynamical XPS experiment, the sample is illuminated with X-rays and a charge compensating low energy electron beam. The sample substrate is subjected to a time varying external stimulus $V(t)$. In this simplified model, the surface potential of each domain is described by a lumped $R$ and $C$ circuit, where the X-ray-induced photoemission currents and electron gun currents are lumped into a single voltage controlled current source. Due to different dynamic and static solutions of the surface potential, domains with different effective $R$ and $C$ values will have different time varying surface potentials. The surface of the domains are assumed to be electrically isolated. Alternatively, the $R$ and $C$ values of the domains can be modulated in time, for example by shining an amplitude modulated external light beam with a wavelength tuned to the absorption of a particular domain. Further models for layered multiple domains can also be derived from the presented model in a straight forward manner. The resistance values $R_{n}$ for each domain are assumed to be linear, although conduction in dielectric materials generally have highly nonlinear behavior $[23,24]$. Still, for the case of a periodic voltage modulation, a linear resistor approximation is observed to produce good results. The electron gun and the photoelectron currents are modeled by voltage controlled current sources. The nonlinear voltage-current curve $I_{s}\left(V_{s}\right)$ can be extracted through analysis of a series of XPS measurements by applying DC voltages $V_{D C}$ to a silicon sample through a series resistance $R_{\text {ext }}$, a value typically around $1 \mathrm{M} \Omega$.

For such static measurements, the binding energy of the biased substrate peak (typically gold) will shift by $V_{s}$ with respect to the true binding energy that can be directly related to the lumped external current by

$I_{s}\left(V_{s}\right)=\frac{V_{s}-V_{D C}}{R_{e x t}}$.

The current-voltage relation dependence $I_{S}\left(V_{S}\right)$ is assumed to be the same throughout the dynamic measurements. The electron gun behaves like a vacuum diode and the typical $I_{s}\left(V_{s}\right)$ can be approximated by a piecewise linear curve, where for negative $V_{s}$ the current is nearly constant, dominated by the photoelectron current and for positive $V_{s}$ (above a threshold value that depends on the electron beam energy) the electron current proportional to $V_{s}$ dominates.

The excitation voltage $V(t)$ can be an arbitrary time varying voltage. Consider a single domain with given $R$ and $C$ values. In order to calculate the XPS spectra collected under such conditions, we need to solve the differential equation describing the dynamics of the surface potential $V_{s}(t)$ given by

$C \frac{d\left(V_{s}-V\right)}{d t}+\frac{V_{s}-V}{R}+I_{s}\left(V_{s}\right)=0$.

Solutions to Eq. (2) can be obtained by numerical integration for arbitrary excitations $V(t)$. For a periodic excitation of period $T$, one can calculate a line-shape function that gives the intensity of the XPS signal at a voltage shift of $v$ as

$g(v)=\frac{1}{T} \int_{0}^{T} \delta\left[V_{s}(t)-v\right]$.

The significance of the line-shape function can be understood intuitively as follows: $g(v)$ quantifies the time spent by the surface potential $V_{s}(t)$ in the vicinity of the potential $v$. It can be seen that $\int_{-\infty}^{\infty} g(v) d v=1$ and hence the line-shape function is normalized, meaning the surface potential $V_{s}(t)$ must be at a value between $-\infty$ and $\infty$ during the period. In a numerical solution of Eq. (2), the lineshape can be calculated simply using a histogram of the values of $V_{s}(t)$ at discrete time steps, and subsequent normalization.

The XPS spectrum recorded during external voltage stimulus $S_{X P S}\left(V_{B E}\right)$ can now be calculated by convolution of the line-shape function $g(v)$ with the original spectrum $S_{0}\left(V_{B E}\right)$ obtained for a zero surface potential shift as

$S_{X P S}\left(V_{B E}\right)=\int_{-\infty}^{\infty} g\left(V_{B E}-\lambda\right) S_{0}(\lambda) d \lambda$.

In reality, the model predicts that, even if the external bias is zero, due to the finite resistance of the surface to the substrate, there may be charging and the surface potential of $V_{s}$ may be shifted from zero. If we assume that the external current sources are identical for each domain, this problem can be solved by applying a bias where there is net zero surface current. Also, the relative intensities of the X-ray source and the electron gun can be adjusted to coincide this condition with zero external bias.

In the following sections, we analyze the properties of lineshapes calculated in the described manner for various excitation waveforms and features of XPS spectra obtained under such excitations.

\section{Square wave excitation}

In this section, a periodic square wave potential is assumed to be applied to the substrate. The potential $V(t)$ is described by $V(t)=V_{p}$ for $0<t-n T<T / 2$ and $V(t)=V_{m}$ for $T / 2<t-n T<T$ 


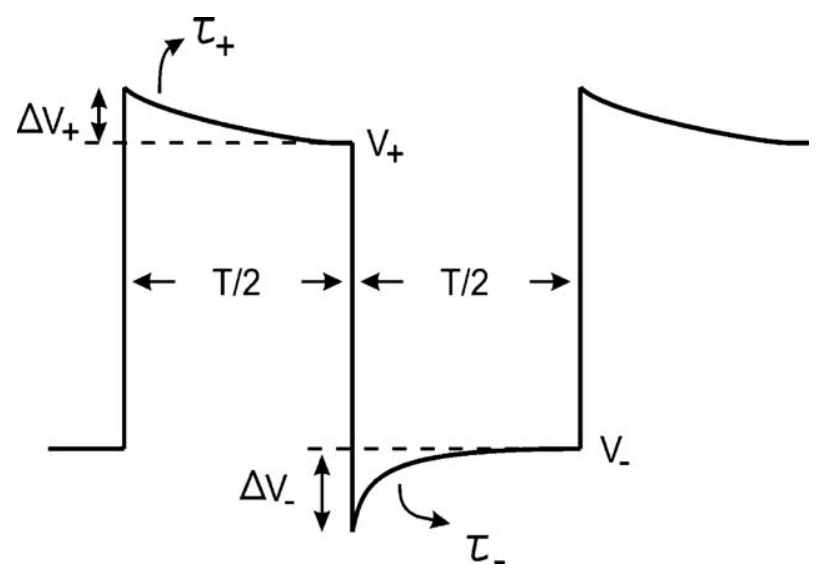

Fig. 2. The time varying surface potential $V_{s}(t)$ under square wave excitation can be approximated by two exponential decays. The time constants $\tau_{+}$and $\tau_{-}$are different for the two half-periods due to changes in the equivalent small-signal shunt resistance of the current source.

where $n$ is an integer and $T=1 / f$ is the period. This waveform is particularly suitable since typical surface potentials with respect to the substrate are on the order of few volts in a real experiment, and by choosing $V_{m}=-10 \mathrm{~V}$ and $V_{p}=10 \mathrm{~V}$, the surface potentials lay in two linear regions of the $I_{s}\left(V_{s}\right)$ curve. In such a case, piecewise analytical solutions can be given for $V_{s}(t)$ and calculation of the lineshapes and resulting spectra can be rapidly performed without numerically solving Eq. (2).

When the sample is excited by a square wave, the surface potential can be piecewise described by exponential decays typical of a first order circuit (Fig. 2). If $V_{m} \simeq-10 \mathrm{~V}$ and $V_{p} \simeq 10 \mathrm{~V}$, the two half-periods generally have different time constants. The reason for this is that the slope of the $I_{S}\left(V_{s}\right)$ curve is different for the two half-periods, and the voltage controlled current $I_{s}\left(V_{s}\right)$ acts as a small-signal resistance in parallel with the sample resistance. In the steady state, the surface voltages for the positive and negative half-periods can be written as $V_{s}(t)=V_{+}+\Delta V_{+} \exp \left(-t / \tau_{+}\right)$and $V_{s}(t)=V_{-}+\Delta V_{-} \exp \left(-(t-T / 2) / \tau_{-}\right)$. Here, $V_{+}, V_{-}, \Delta V_{+}$and $\Delta V_{-}$ are unknowns to be determined. The equations needed to solve for the unknowns can be obtained from the requirements of continuity of voltages at the transitions $(t=n T$ and $t=n T+T / 2, n$ being an integer) and from asymptotic values resulting from the steady state assumption. The time constants are $\tau_{-} \cong\left(R \| R_{E}^{-}\right) C$ and $\tau_{+} \cong\left(R \| R_{E}^{+}\right) C$, where $R_{E}^{+}=\left.\left[d V_{s} / d I_{s}\right]\right|_{V_{s} \sim V_{p}}$ and $R_{E}^{-}=\left.\left[d V_{s} / d I_{s}\right]\right|_{V_{s} \sim V_{m}}$ is the equivalent small-signal resistance of the electron gun. The solution can be expressed as

$\Delta V_{+}=-\frac{\left[\left(V_{+}-V_{-}\right)-V_{\text {pulse }}\right]\left(1-\exp \left(-T / 2 \tau_{-}\right)\right)}{1-\exp \left(-T / 2 \tau_{-}\right) \exp \left(-T / 2 \tau_{+}\right)}$

$\Delta V_{-}=-\left[\left(V_{+}-V_{-}\right)-V_{\text {pulse }}+\Delta V_{+} \exp \left(-T / 2 \tau_{+}\right)\right]$

where $V_{\text {pulse }}=V_{p}-V_{m}$. The values of $V_{+}$and $V_{-}$can be determined by solving for the asymptotic equilibrium conditions $\left(V_{+}-V_{p}\right) / R+I_{s}\left(V_{+}\right)=0$ and $\left(V_{-}-V_{m}\right) / R+I_{s}\left(V_{-}\right)=0$.

The lineshape $g(v)$ can be calculated using the above solution. When lineshapes calculated by direct numerical integration of Eq. (2) and the analytical expressions given above are compared for a typical experimental $I_{S}\left(V_{S}\right)$, it is seen that both approaches produce nearly identical results for a wide range of parameters (frequency $f$ and sample properties $R$ and $C$ ).

In order to illustrate the lineshapes under square wave excitation, we plot the lineshapes for typical experimental values of $R=5 \mathrm{M} \Omega$ and $R=10 \mathrm{M} \Omega, \quad C=100 \mathrm{nF}, \quad V_{p}=10 \mathrm{~V}$ and $V_{m}=-10 \mathrm{~V}$ for different frequencies of $f=0.1 \mathrm{~Hz}, 1 \mathrm{~Hz}$ and $10 \mathrm{~Hz}$ as shown in Fig. 3.
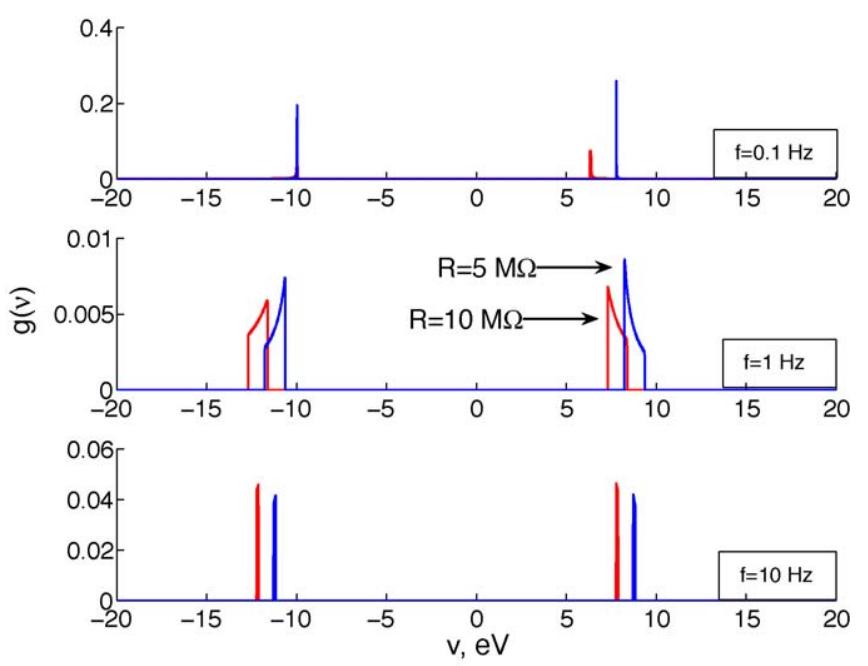

Fig. 3. Lineshapes $g(v)$ for typical experimental values of $R=5 \mathrm{M} \Omega$ and $R=10 \mathrm{M} \Omega$, $C=100 \mathrm{nF}$, for a square wave excitation with $V_{p}=10 \mathrm{~V}$ and $V_{m}=-10 \mathrm{~V}$ for different frequencies of $f=0.1 \mathrm{~Hz}, 1 \mathrm{~Hz}$ and $10 \mathrm{~Hz}$.

A more detailed behavior of the lineshapes can be seen if $g(v)$ is plotted in a gray scale image as a function of frequency as shown in Fig. 4.

Figs. 3 and 4 present typical behavior of peaks observed in a pulsed XPS experiment. A single peak will divide into two peaks with shifts corresponding to the two peaks of $g(v)$, and have an excess broadening when the time constant $\tau \simeq R C$ is close to $T / 2$. In the high an low frequency limits, the peaks will be sharp, with no or little excess broadening. The positions of the positive and negative shifted peaks will reach steady state values in the low and high frequency limits, and a transition will occur between the two steady state values when $T / 2 \simeq R C$. In order to quantify these features, we define the average positions of the positive and negative peaks and their variances as $V_{a, p}=\left.\langle g(v) v\rangle\right|_{v>0}$, $V_{a, n}=\left.\langle g(v) v\rangle\right|_{\nu<0}, \quad \Delta V_{p}=\sqrt{\left.\left\langle g(v)\left(v-V_{a, p}\right)^{2}\right\rangle\right|_{\nu>0}}$ and $\Delta V_{n}=$ $\sqrt{\left.\left\langle g(v)\left(v-V_{a, n}\right)^{2}\right\rangle\right|_{\nu<0}}$. A binding energy difference can be defined

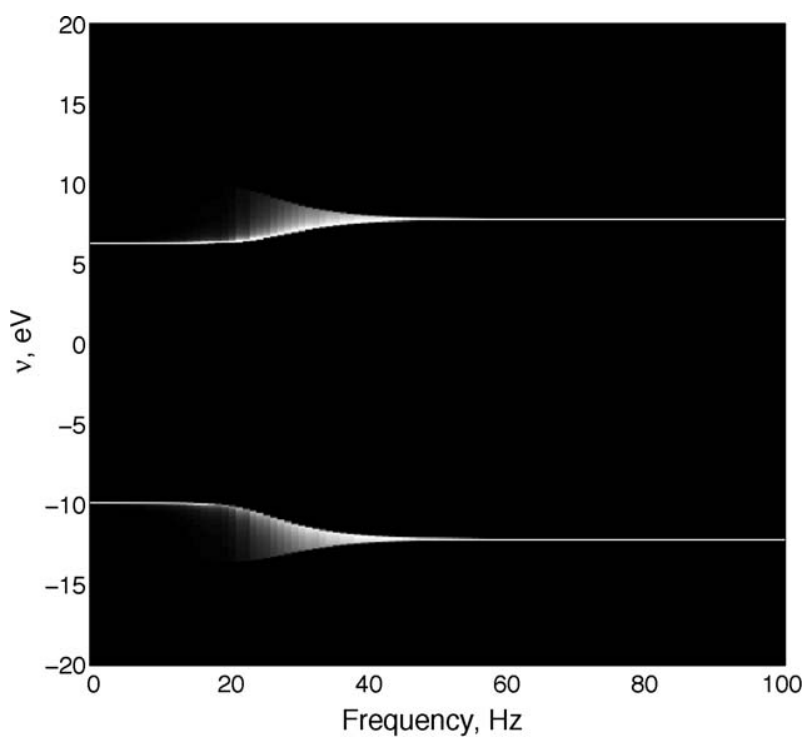

Fig. 4. Lineshapes $g(v)$ for a square wave excitation with $V_{p}=10 \mathrm{~V}$ and $V_{m}=-10 \mathrm{~V}, R=10 \mathrm{M} \Omega$ and $C=1 \mathrm{nF}$, plotted in a gray scale image as a function of frequency $(g(v)$ 's are normalized to maximum value for each frequency for better figure clarity. Originally, during the broadening around $25 \mathrm{~Hz}$, peak intensities decrease). 

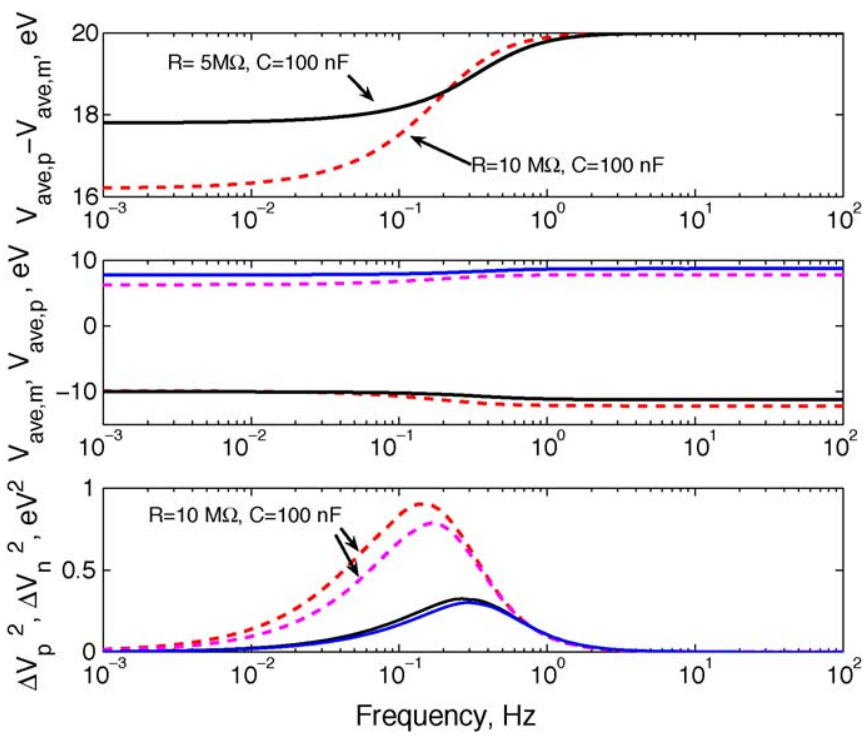

Fig. 5. Variables $B E D=V_{a, p}-V_{a, n}, V_{a, p}, V_{a, n}, \Delta V_{p}$ and $\Delta V_{n}$ are plotted for $R=5 \mathrm{M} \Omega$ and $R=10 \mathrm{M} \Omega, C=100 \mathrm{nF}$ as a function of frequency (see text for definitions of the variables).

as $B E D=V_{a, p}-V_{a, n}$. In the low frequency limit, the effect of the electron gun current is to reduce the $B E D=V_{a, p}-V_{a, n}$ to a value lower than $V_{\text {pulse }}$. In the high frequency limit, capacitance dominates the response and $B E D \simeq V_{\text {pulse }}$. After making these definitions, now behavior of XPS peaks belonging to domains of certain electrical properties can be compared more quantitatively. As an example, the defined variables $B E D, V_{a, p}, V_{a, n}, \Delta V_{p}$ and $\Delta V_{n}$ are plotted for $R=5 \mathrm{M} \Omega$ and $R=10 \mathrm{M} \Omega, C=100 \mathrm{nF}$ as a function of frequency as shown in Fig. 5. The actual half width of the positive shifted XPS peak with natural half width of $\Delta V_{0}$ can be calculated as $\Delta V_{+} \simeq \sqrt{\Delta V_{0}^{2}+\Delta V_{p}^{2}}$. Same applies for the negative shifted peak $\Delta V_{-} \simeq \sqrt{\Delta V_{0}^{2}+\Delta V_{n}^{2}}$.

The effect of a square wave excitation with a duty-cycle other than $50 \%$ is also worth mentioning. Consider a case where $V_{m}=0$, $V_{p}=10 \mathrm{~V}$ and the duration of the positive voltage pulse is relatively small with respect to the period. This excitation is approximately an impulse train excitation. In such a case, the relative intensities of the positive and negative shifted peaks are not equal, since the surface potential is rapidly shifted by the impulse towards positive voltages, and without having time to relax, is again shifted back to its original position. During the pulse, electrons are attracted towards the surface and the capacitor is negatively charged. During the time where the excitation is $V(t)=V_{m}=0$, the surface charge relaxes through tunneling into the substrate or through compensation by escaping photoelectrons. When the time constant of this relaxation $(\tau \simeq R C)$ is similar to the period, broadening of the peak becomes maximum. Also, due to the small amount of time spend at the positive voltage pulse duration, doubling of the peak becomes insignificant. Also, a small shift of the peaks dependent on their effective $R$ and $C$ values is observed. The broadenings are, however, relatively small compared to natural line widths of common XPS peaks.

\section{Small amplitude square wave excitation with a DC offset}

It is interesting to investigate the effect of a square wave excitation with a DC offset and a small amplitude, i.e. where $V_{m}=$ $V_{D C}-\delta / 2$ and $V_{p}=V_{D C}+\delta / 2$. Here $V_{D C}$ is the DC offset and $\delta$ is the
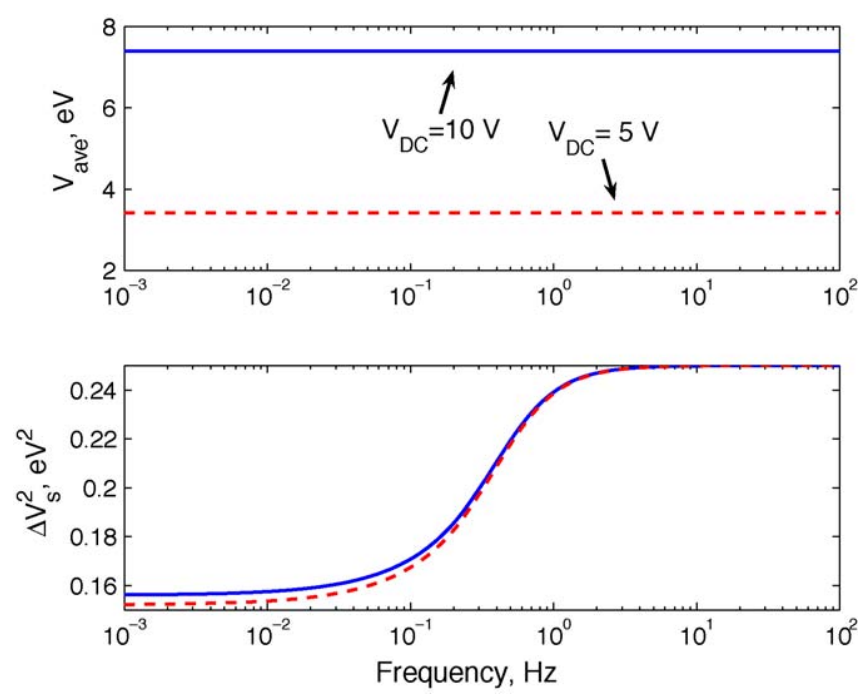

Fig. 6. Variables $V_{a, s}$ and $\Delta V_{s}$ are plotted for $R=5 \mathrm{M} \Omega, C=100 \mathrm{nF}$ as a function of frequency for $V_{D C}=5 \mathrm{~V}$ and $V_{D C}=10 \mathrm{~V}$ and $1 \mathrm{~V}$ peak-to-peak modulation amplitude (see text for definitions of the variables). The frequency does not significantly affect the peak position, however the broadening of the peaks change as the frequency is swept from DC to above the cut-off frequency of the RC filter formed by the domain.

amplitude of the square wave. The significance of such an excitation is that it includes the effect of just applying a DC voltage to the sample, which results in shifts of peaks proportional to the resistance, and also the effect of applying a modulation to the substrate, resulting in broadening of the peaks when the time constants match the modulation frequency. Moreover, by proper choice of $\delta$, doubling of peaks or smearing of different peaks can be avoided. The model described in the previous section describes this case as well. It is convenient to define $V_{a, s}=\left(V_{a, p}+V_{a, n}\right) / 2$, which denotes the average surface potential, and $\Delta V_{s}=$ $\sqrt{\left\langle g(v)\left(v-V_{a, s}\right)^{2}\right\rangle}$ which denotes the total broadening of the peak. Numerically solving Eq. (2), the resulting average peak shift and variance are plotted in Fig. 6 for $R=5 \mathrm{M} \Omega, C=100 \mathrm{nF}$ and as a function of frequency for different $V_{D C}$ and $\delta=1 \mathrm{~V}$. It is seen that the frequency does not significantly affect the peak position, however the broadening of the peaks becomes greater as the
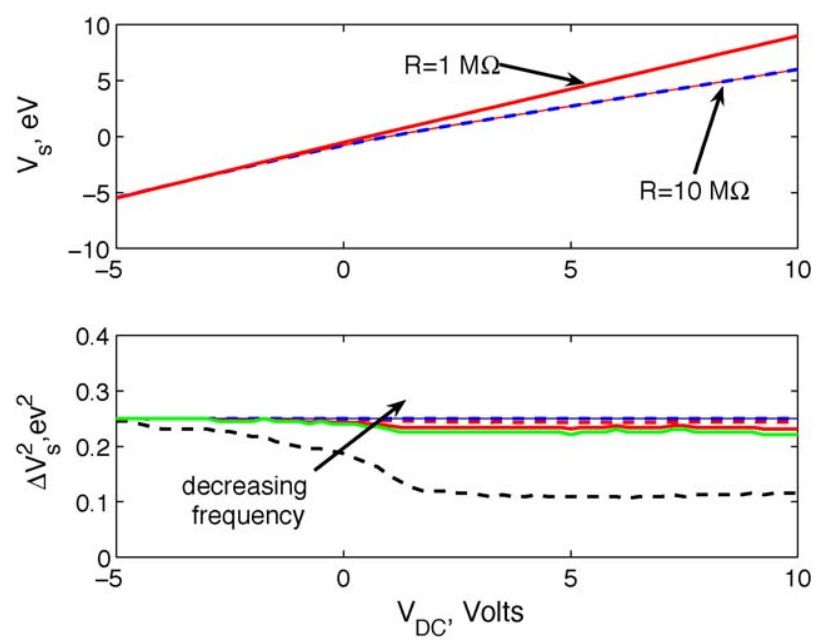

Fig. 7. Variables $V_{a, s}$ and $\Delta V_{s}$ are plotted for $R=5 \mathrm{M} \Omega$ (solid) and $R=10 \mathrm{M} \Omega$ (dashed), $C=100 \mathrm{nF}$ as a function $V_{D C}$ for $f=0.01 \mathrm{~Hz}, 1 \mathrm{~Hz}$ and $100 \mathrm{~Hz}$. The peak positions are shifted by applying a DC, and the broadening of the peaks have a slight dependence on bias due to changing slope of the $I_{s}\left(V_{s}\right)$ curve as a function $V_{D C}$. 

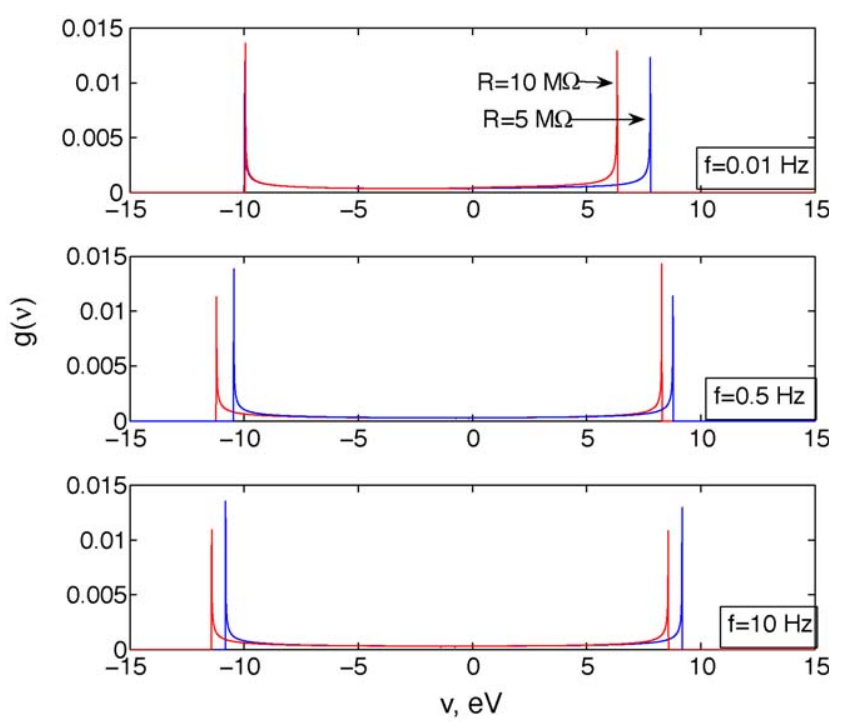

Fig. 8. Lineshapes obtained for a sinusoidal modulation with $V(t)=V_{a} \cos (2 \pi f t)$ for $R=5 \mathrm{M} \Omega, C=100 \mathrm{nF}$ and $f=0.01 \mathrm{~Hz}, 0.5 \mathrm{~Hz}$ and $100 \mathrm{~Hz}$. Due to the nature of excitation, lineshapes are very broad and this causes excessive smearing of the XPS peaks.

frequency is above the cut-off frequency of the RC filter formed by the domain. In order to show the effect of $V_{D C}$, same variables are plotted in Fig. 7 as a function of $V_{D C}$.

The modulation scheme discussed here has the nice feature that, the peaks do not split into two well separated peaks but stay in the vicinity of the original peak, therefore making it easier to analyze samples with multiple peaks. The DC voltage can be used to separate the peaks with different resistance values, and broadening of the peaks can be used to characterize the time constant. Also, the additional parameter introduced, namely the modulation amplitude, provides another degree of freedom for data analysis.

\section{Sinusoidal excitation}

In order to give a more complete picture of the effect of bias modulation on XPS spectra, a sinusoidal modulation is also considered. A sinusoid has a single frequency component, and it is interesting to see if such a modulation has any significant benefits over square wave excitations. In this case, the excitation is of the form $V(t)=V_{a} \cos (2 \pi f t)$. Using the numerical solution to Eq. (2) for such an excitation, lineshapes are plotted in Fig. 8 for $R=5 \mathrm{M} \Omega$ and $R=10 \mathrm{M} \Omega, C=100 \mathrm{nF}$ for $f=0.01 \mathrm{~Hz}, 0.5 \mathrm{~Hz}$ and $10 \mathrm{~Hz}$.

It is seen in Fig. 8, that due to the nature of excitation, lineshapes are very broad with nonzero values in between the two extremes and this causes excessive smearing of the XPS peaks. If a sinusoidal excitation with a DC offset and small amplitude is used, i.e. $V(t)=V_{D C}+V_{a} \cos (2 \pi f t)$, resulting lineshapes become similar to those shown in the previous section.

\section{Conductivity modulation}

Another type of modulating the surface potential can be possible, by modulating the effective resistance value through external means. One interesting example of such as a modulation can be achieved by shining a modulated light beam onto a semiconductor sample. In such a case, due to absorption of light, carriers are generated and conductivity is modulated. By choosing the wavelength of light to coincide with absorption peak of the sample, a combined form of XPS and optical
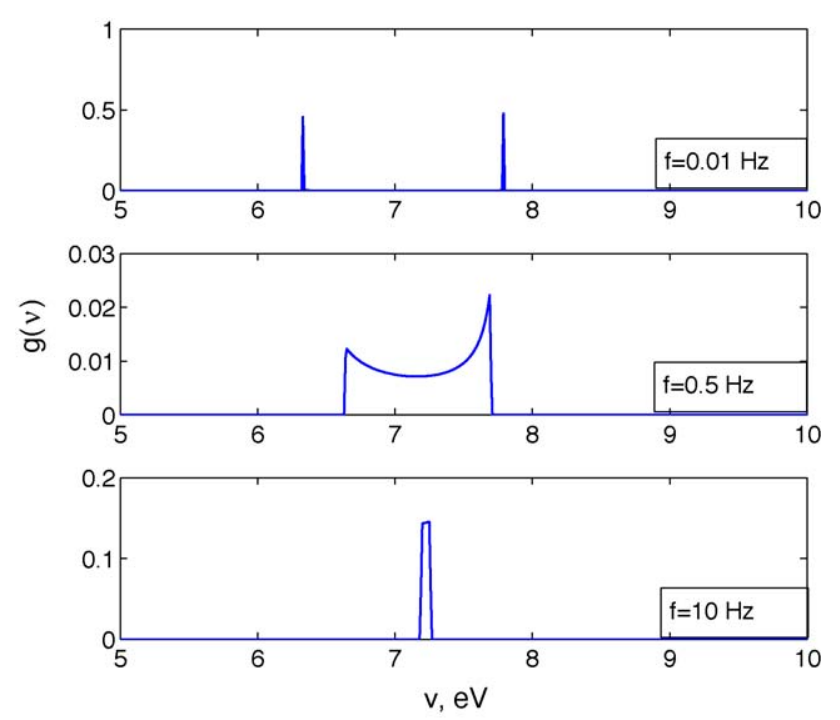

Fig. 9. Lineshapes obtained for a square wave modulation of the resistance for $R=10 \mathrm{M} \Omega, C=100 \mathrm{nF}, f=0.01 \mathrm{~Hz}, 0.5 \mathrm{~Hz}$ and $100 \mathrm{~Hz}, V_{D C}=10 \mathrm{~V}$, for different modulation fraction of $\alpha=0.5$.

absorption spectroscopy can be performed. This type of dynamic XPS spectroscopy may also be suitable for systems where only a DC bias is available and the sample can be accessed only optically through a window.

Assume, the resistance value $R$ is not constant but is a function of time $R=R(t)$ and a fixed DC bias $V_{D C}$ is applied to the substrate. The function $R(t)$ is assumed to be a square wave and $R(t)=R$ for $0<t-n T<T / 2$ and $R(t)=\alpha R$ for $T / 2<t-n T<T$ where $n$ is an integer and $T=1 / f$ is the period. In this case, the lineshapes can be calculated again by solving Eq. (2), and results are plotted for $R=10 \mathrm{M} \Omega, \quad C=100 \mathrm{nF}, \quad V_{D C}=10 \mathrm{~V}, \alpha=0.5$, for $f=0.01 \mathrm{~Hz}$, $0.5 \mathrm{~Hz}$ and $10 \mathrm{~Hz}$ as shown in Fig. 9.

The peak position and variance of the lineshapes are also plotted as shown in Fig. 10.
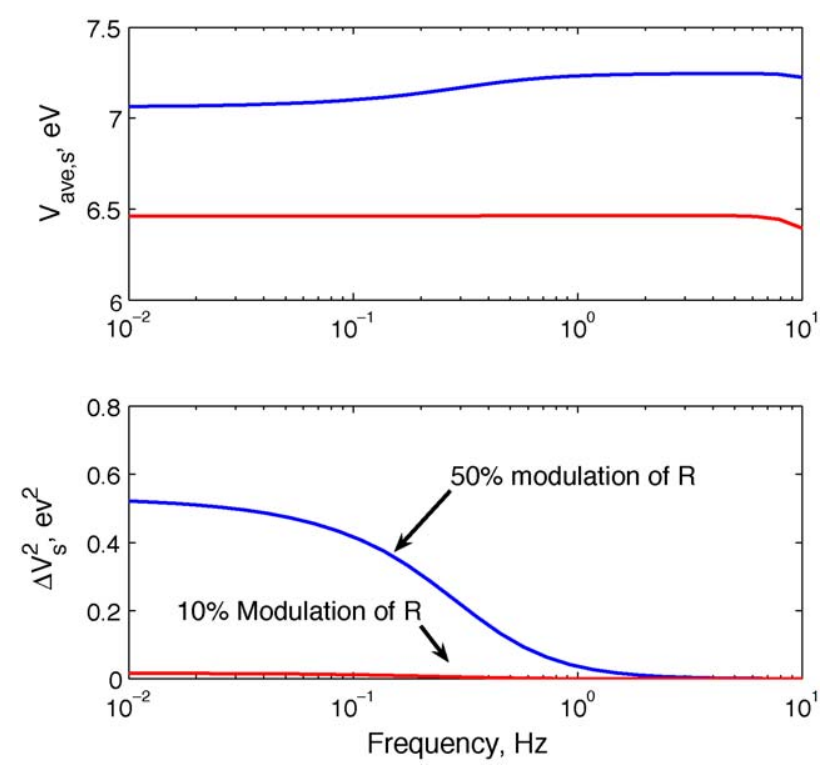

Fig. 10. Average peak shifts and variances of lineshapes are plotted for a square wave modulation of the resistance as a function of frequency for different modulation fractions of $\alpha=0.5$ and 0.9 (50\% and $10 \%$ modulation depths respectively). Parameters are $R=10 \mathrm{M} \Omega, C=100 \mathrm{nF}$ and $V_{D C}=10 \mathrm{~V}$. 


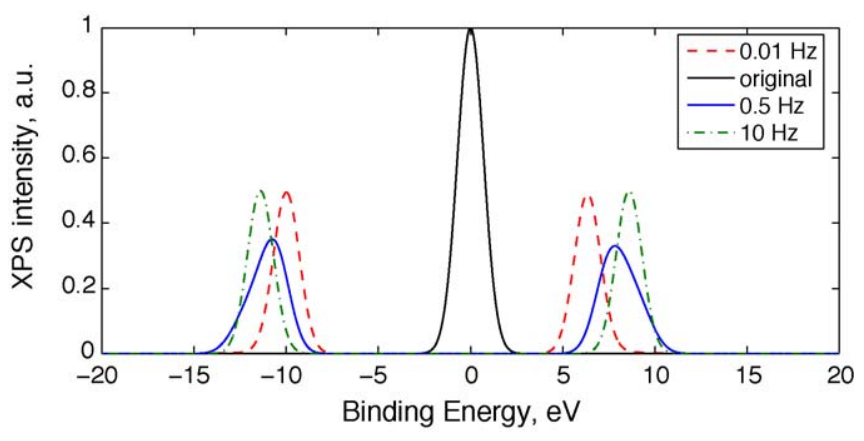

Fig. 11. Calculated spectra demonstrate frequency dependent changes in peak positions and intensities, as well as changes in peak shapes such as broadening and enhanced skewness in the case of a large amplitude symmetric square wave excitation. Parameters are $R=10 \mathrm{M} \Omega, C=100 \mathrm{nF}$ and $V_{p}=10 \mathrm{~V}, V_{m}=-10 \mathrm{~V}$.

\section{Simulation of XPS spectra using the lineshapes}

Once the line-shape function $g(v)$ is calculated for a specific modulation scheme, the XPS spectra can be calculated using the convolution in Eq. (4), and using XPS data obtained without modulation and zero net charging conditions. Although, once the lineshapes are calculated, it is mathematically straightforward to calculate the XPS spectra under modulation of the bias or resistance, for the sake of better intuition we plot exemplary calculated spectra for the mentioned modulation schemes. Assuming a Gaussian shaped peak with the form $S_{0}(v)=\exp \left(-v^{2}\right)$, calculated spectra for a large amplitude square wave excitation $\left(V_{p}=10 \mathrm{~V}, V_{m}=-10 \mathrm{~V}\right)$ is plotted as shown in Fig. 11 for $R=10 \mathrm{M} \Omega, C=100 \mathrm{nF}$. Corresponding lineshapes are similar to those seen in Fig. 3.

It can be seen that, when the period of the excitation is close to the time constant, the peak is broadened and becomes asymmetric. As a result of the broadening, as the area under the curve must be constant in this configuration, the peak intensity is reduced. Using the well known property of convolutions, this reduction can be calculated approximately using $I_{\text {peak }}^{\prime} / I_{\text {peak }}^{0} \simeq \sqrt{\sigma_{0}^{2} /\left(\sigma_{0}^{2}+\sigma_{L S H}^{2}\right)}$, where $\sigma_{0}$ is the variance of the original peak and $\sigma_{L S H}$ is the variance of the line-shape function (denoted by $\Delta V_{s}$ in previous sections).

This reduction of peak intensity is easier to observe without going to data analysis and proves useful especially in the case of a small amplitude square wave excitation with a DC bias (Fig. 12). For this case, below the cut-off frequency, broadening is reduced (see Fig. 7) and peak intensity is increased.

In order to demonstrate the effect of sinusoidal large amplitude modulation, we plot calculated spectra for a sinusoidal modulation with $10 \mathrm{~V}$ amplitude as shown in Fig. 13.

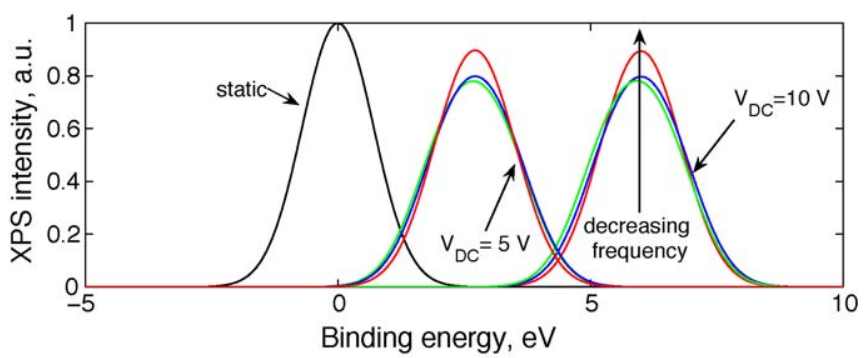

Fig. 12. Calculated spectra demonstrate frequency dependent changes in peak positions and intensities, in the case of a small amplitude square wave excitation with a DC bias. Parameters are $R=10 \mathrm{M} \Omega, C=100 \mathrm{nF}$ and $V_{p}-V_{m}=1 \mathrm{~V}, V_{p}=5 \mathrm{~V}$ and $10 \mathrm{~V}$.

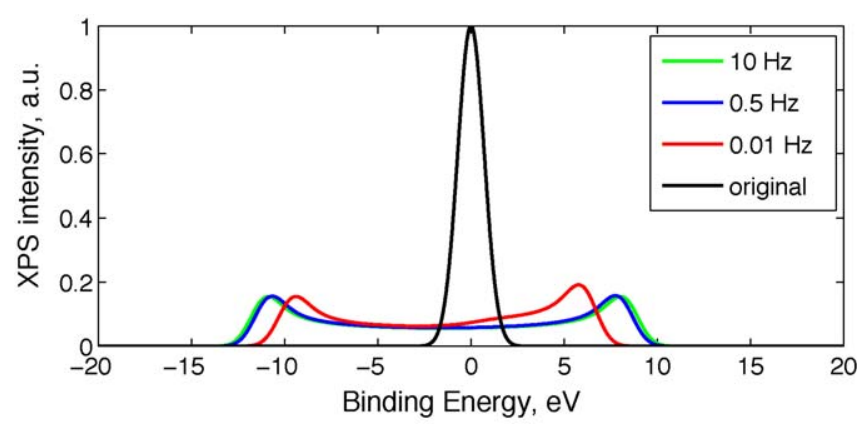

Fig. 13. Calculated spectra demonstrate the smearing effect of a large amplitude sinusoidal excitation. Parameters are $R=10 \mathrm{M} \Omega, C=100 \mathrm{nF}$ and $V_{a}=10 \mathrm{~V}$.

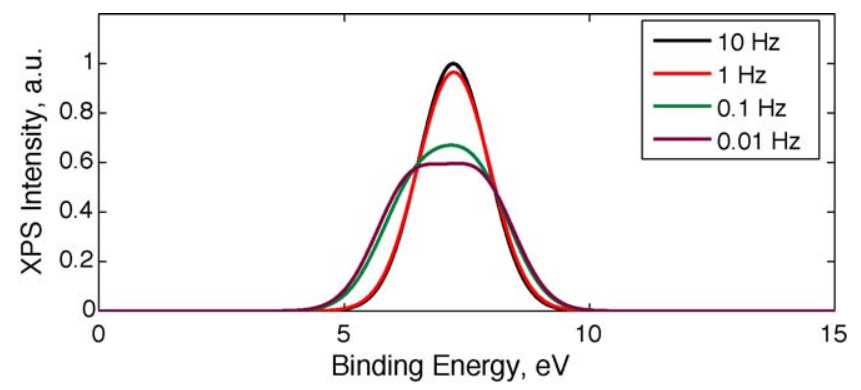

Fig. 14. Calculated spectra for an unperturbed Gaussian peak of the form $S_{0}(v)=$ $\exp \left(-v^{2}\right)$ demonstrate the effect of a modulation of the resistance $R$ by an external mean, such as shining modulated light onto a semiconducting layer. Parameters are $R=10 \mathrm{M} \Omega, C=100 \mathrm{nF}, V_{D C}=10 \mathrm{~V}$, and $\alpha=0.5$ (corresponding to $50 \%$ resistance modulation). Below the average cut-off frequency, peak shifts and broadens. Broadening results in reduction of the peak intensity.

It can be seen that, for large amplitude sinusoidal case, there is a large smearing of the peaks. This smearing potentially results in intermixing of closely separated peaks, making interpretation difficult.

Conductivity modulation is fundamentally different from the above described voltage modulations in nature. At high frequencies of modulation of the resistance R, the RC circuit filters out the effect and broadening is less significant. Below the cut-off frequency, system begins to behave as one with two different systems with different $R$ values, so the lineshape splits into two well separated peaks. The effect of this is to introduce a broadening, a peak shift and a reduction in peak intensity as shown in Fig. 14.

\section{Conclusions}

In the previous sections, different modulation schemes have been investigated for their effect on the shifts and broadenings of XPS peaks belonging to different electrical domains. It is seen that, large amplitude square wave and small-signal square wave modulation can be used as voltage modulation schemes, without resulting in smear XPS spectra. Small-signal square wave excitation with a DC offset is particularly useful since doubling of the peaks can be avoided and there is an additional degree of freedom, the small-signal amplitude. In general, when a square wave modulation is applied, in the low frequency or high frequency limits, the shifts reach asymptotic values and broadenings are reduces, and near the characteristic frequency of the RC circuit, the shifts show a transition between the two asymptotic values and broadening reaches a maximum. Sinusoidal modulation was also investigated and it was seen that a large amplitude sinusoidal excitation causes excessive smearing of peaks, which complicates 
interpretation. A small-signal sinusoidal excitation with a DC offset becomes in effect similar to a square wave.

In this model, it is assumed that individual domains do not affect each other's electrical properties. Under this assumption, an XPS peak having contributions from domains with different electrical properties can be analyzed by analyzing and adding the contributions of different components. Small-signal square wave excitation can be used to separate the peaks in binding energy by making measurements at different DC offsets, and simultaneously the time constants can be measured.

Another important modulation scheme investigated is the modulation of the resistance of the surface using an external mean, such as by shining modulate light intensity on a semiconductor. As the wavelength and intensity of light are free parameters, light absorption and conductivity modulation of domains on surfaces may be investigated with chemical specificity using a properly designed sample and the described modulation scheme. A square wave modulation of incident radiation may have an observable effect for a sample whose equivalent resistance changes with illumination, since a suddenly reduced resistance will cause a domain absorbing the illumination to experience an electrical short circuit to the ground plane, and this may result in a large shift of the peak with minimal excess broadening. In such a case, there will be a doubling of the peaks in the spectra, especially if the resistance modulation is strong. In the case of small modulation depth of the resistance, a broadening of the peak can be expected, accompanied by an apparent intensity reduction. One interesting feature of this sort of modulation is that, it has effects similar to a small-signal voltage modulation in terms of broadening, however has inverse dependence on the illumination modulation frequency.

In summary, we have discussed the implications of the surface charging model on XPS spectra collected under modulated sample bias or resistance. Extension to modulations of capacitance through a modulation of the dielectric constant of the sample, is straight forward. The model allows investigation of arbitrary modulation schemes. Modulation becomes especially useful when distinguishing rare components of composite surfaces.

\section{Acknowledgements}

The author gratefully thanks Şefik Süzer for introducing him to the topic as well as for valuable discussions on the nature of experimental results. This work was partially funded by the State Planning Organization of the Turkish Republic, Project UNAM.

\section{References}

[1] I. Lindgren, J. Elec. Spec. Rel. Phenom. 137 (140) (2004) 59.

[2] N.H. Turner, J.A. Schreifels, Anal. Chem. 66 (12) (1994) 163.

[3] M.P. Seah, Surf. Int. Anal. 2 (6) (2004) 222.

[4] F.J. Grunthaner, P.J. Grunthaner, R.P. Vasquez, B.F. Lewis, J. Maserjian, A. Madhukar, Phys. Rev. Lett. 43 (1979) 1683.

[5] M.J. Guittet, J.P. Crocombette, M. Gautier-Soyer, Phys. Rev. B 63 (2001) 125117.

[6] K. Endo, S. Koizumi, T. Otsuka, M. Suhara, T. Morohasi, E.Z. Kurmaev, D.P. Chong, J. Comput. Chem. 22 (1) (2000) 102.

[7] A. Nikitin, H. Ogasawara, D. Mann, R. Denecke, Z. Zhang, H. Dai, K. Cho, A. Nilsson, Phys. Rev. Lett. 95 (2005) 225507.

[8] A. Dane, U.K. Demirok, A. Aydinli, S. Suzer, J. Phys. Chem. B 110 (3) (2006) 1137.

[9] I.B. Akca, A. Dana, A. Aydinli, R. Turan, Appl. Phys. Lett. 92 (2008) 052103.

[10] S. Suzer, Anal. Chem. 75 (2003) 7026-7029.

[11] F. Karadas, G. Ertas, S. Suzer, J. Phys. Chem. B 108 (2004) 1515-1518.

[12] G. Ertas, S. Suzer, Surf. Interface Anal. 36 (2004) 619-623.

[13] U.K. Demirok, G. Ertas, S. Suzer, J. Phys. Chem. B 108 (2004) 5179-5181.

[14] G. Ertas, U.K. Demirok, S. Suzer, Appl. Surf. Sci. 248 (2005) 12-15.

[15] G. Ertas, U.K. Demirok, A. Atalar, S. Suzer, Appl. Phys. Lett. 86 (2005) 183110.

[16] E. Karabudak, U.K. Demirok, S. Suzer, Surf. Sci. Lett. 600 (2006) L12-L14.

[17] G. Barth, R. Linder, C. Bryson, Surf. Int. Anal. 11 (6) (1988) 307.

[18] P.E. Larson, M.A. Kelly, J. Vac. Sci. Technol. A 16 (6) (1998) 3483.

[19] S. Suzer, A. Dana, J. Phys. Chem. B 110 (2006) 19112.

[20] S. Suzer, A. Dana, G. Ertas, Anal. Chem. 79 (2007) 183.

[21] H. Sezen, G. Ertas, A. Dana, S. Suzer, Macromolecules 40 (12) (2007) 4112.

[22] S. Suzer, H. Sezen, A. Dana, Anal. Chem. 80 (2008) 3931.

[23] D.J. DiMaria, J.W. Stasiak, J. Appl. Phys. 65 (1989) 2342.

[24] D.J. DiMaria, D.W. Dong, C. Falcony, T.N. Theis, J.R. Kirtley, J.C. Tsang, D.R. Young, F.L. Pesavento, S.D. Brorson, J. Appl. Phys. 54 (1983) 5801. 